\title{
BUBBLE FORMATION FROM WALL ORIFICE IN LIQUID CROSS-FLOW UNDER LOW GRAVITY
}

\author{
Henry K. Nahra* \\ Microgravity Science Division, Microgravity Fluid Physics Branch \\ NASA-John H. Glenn Research Center at Lewis Field \\ 21000 Brookpark Rd., MS:500-102 \\ Cleveland, $\mathrm{OH} 44135$ \\ e-mail:henry.k.nahra@grc.nasa.gov \\ Fax:(216) 4338050 \\ and \\ Y. Kamotani \\ Department of Mechanical \& Aerospace Engineering \\ Case Western Reserve University, \\ Cleveland, $\mathrm{OH} 44106$
}

\begin{abstract}
Two-phase flows present a wide variety of applications for spacecraft thermal control systems design. Bubble formation and detachment is an integral part of the twophase flow science. The objective of the present work is to experimentally investigate the effects of liquid cross-flow velocity, gas flow rate, and orifice diameter on bubble formation in a wall-bubble injection configuration. Data were taken mainly under reduced gravity conditions but some data were taken in normal gravity for comparison. The reduced gravity experiment was conducted aboard the NASA DC-9 Reduced Gravity Aircraft. The results show that the process of bubble formation and detachment depends on gravity, the orifice diameter, the gas flow rate, and the liquid cross-flow velocity. The data are analyzed based

- Corresponding author
\end{abstract}



on a force balance, and two different detachment mechanisms are identified. When the gas momentum is large, the bubble detaches from the injection orifice as the gas momentum overcomes the attaching effects of liquid drag and inertia. The surface tension force is much reduced because a large part of the bubble pinning edge at the orifice is lost as the bubble axis is tilted by the liquid flow. When the gas momentum is small, the force balance in the liquid flow direction is important, and the bubble detaches when the bubble axis inclination exceeds a certain angle.

Key Words: bubble generation, gas-liquid flow, liquid cross-flow, microgravity, scaling. forces.

\section{INTRODUCTION}

The power requirements for current and future space missions are increasing due to rising power demand. More efficient thermal control systems for space application are therefore needed to handle the increasing power requirements. Two-phase flow thermal control systems provide effective thermal transport in spacecraft (Eastman et al. 1984), which is due to the higher latent heat of vaporization of fluids compared to their sensible heat. Two-phase flow phenomena are encountered in space propulsion systems, thermal control of power generation and distribution subsystems, cryogenic transfer and storage systems, life support systems, and systems related to commercial development of space programs (Ostrach 1988).

Dispersed phase bubble or droplet formation and behavior play an important role in twophase flows. Understanding of such a behavior in microgravity is crucial because of its direct implications to the design of two-phase flow systems for large space structure such as 
the space station. Understanding the behavior of dispersed bubble in micro-gravity includes the understanding of bubble formation, detachment and the forces involved in affecting these two processes. Due to the absence of the buoyancy force, detachment of bubbles exhibits some differences in microgravity. One method to help detach forming bubbles is to apply a cross-flow where the induced drag force strongly contributes to the bubble detachment (Kim et al. 1994).

Several investigators have studied the bubble generation and detachment from submerged nozzle under full gravity and in still or moving fluid. Kumar and Kuloor (1970) published an excellent review of the different experiments performed and proposed models to analyze the bubble diameter at detachment in still liquids. In their review, they considered systems of constant gas flow rates, constant pressure, and briefly addressed the liquid cross flow situation. Tsuge et al. (1981) have investigated bubble evolution and detachment in full gravity from an orifice submerged in a flowing liquid and studied the effects of the liquid velocity on bubble diameter. They also presented calculations to predict the bubble diameter at detachment using an empirical detachment criterion. Al-Hayes and Winterton (1981) measured the bubble diameter at detachment into a flowing liquid in a tube and developed a modified surface tension force using the equilibrium contact angle. Kawase and Ulbrecht (1981) developed a model based on force balance on a droplet or a bubble and simulated the drag force by tilting of the nozzle orifice. Marshall and Chudacek (1993) performed ground experiments to measure the bubble detachment diameter under high liquid flow rates and compared his results with theoretical predictions using a force model and average pressure over the bubble surface derived from the potential flow theory. In all of the above-cited 
work, the buoyancy force is the most dominant in inducing detachment of bubbles.

In low gravity, the force of buoyancy is much reduced, and as a result, bubbles can grow larger than the pipe or channel hydraulic diameter, thereby forming a Taylor bubble, especially when produced using small gas flow rates. Pamperin and Rath (1995) have studied the bubble formation from a submerged nozzle in a still fluid under low gravity conditions and found that detachment occurs beyond a critical Weber number which is defined in terms of the gas velocity. The cross flow of liquid assists in the detachment process as was shown by Kim et al. $(1992,1994)$ who developed a theoretical model based on the force balance to predict the bubble diameter at detachment from a nozzle submerged in a cross- and co-flow of liquid. Bhunia et al. (1998) revisited the co-flow calculations and reformulated the problem by including the relative velocity into the inertia term of the forming bubble. The results compared well with experimental data obtained from a low gravity nozzle-injection bubble-generation and detachment experiment performed on the DC-9 Low Gravity Platform. Tsuge et al. (1997) conducted drop-tower tests for gas injection from a submerged nozzle in a cross- and co-flow of liquid, and determined bubble shapes and sizes.

For a constant gas flow rate and under normal gravity, three regimes for bubble generation have been identified, each of which depends on the gas flow rate. They are the quasi-static, dynamic, and turbulent regimes. The first regime occurs at very low gas flow rate less than $1 \mathrm{~cm}^{3} / \mathrm{s}$ whereas the second occurs at much higher flow rates which extend to $10^{4} \mathrm{~cm}^{3} / \mathrm{s}$ for air-water system (Wraith 1971). The last regime takes place when the gas flow rate is very high such that the frequent bubble-coalescence at the nozzle results in forming a gas jet that discharges the gas into the liquid. 
Although bubble generation by submerged nozzle injection has been extensively studied, emphasis has not been given to the problem of wall bubble injection using gas flow rates that fall in the quasi-static regime under low gravity conditions. Wall injection of bubbles differs from nozzle injection in the presence of liquid cross-flow in some important respects. As illustrated in Fig. 1, in the case of wall injection the bubble motion is restricted by the wall. Under certain conditions a part of the bubble surface in the downstream side touches the wall, which means that the bubble is not completely anchored at the orifice. In addition, the way the bubble is attached to the nozzle tip (assuming that the nozzle wall thickness is much smaller than the inner diameter) is different from the way it is attached to the orifice corner. Those differences are not very important when the bubble moves mainly in the direction of injection, as in the case of bubble generation in normal gravity with no or weak liquid crossflow. However, they become important in reduced gravity with a cross-flow as the bubble tends to move in the downstream direction, affecting the bubble formation process significantly.

In the present work, bubble generation and detachment via wall injection is experimentally studied in order to understand the underlying physical process that governs bubble detachment under low gravity conditions and in the presence of liquid cross-flow. The gas flow rates considered in this study fall in the quasi-static regime. Based on the data and scaling analysis, the bubble generation process is delineated.

\section{EXPERIMENTAL SETUP}

\subsection{Ground Flow Loop}


The 1-g ground flow loop, ( $\mathrm{g} \equiv$ Earth gravitational acceleration) was built in order to perform the Bubble Formation and Detachment Normal Gravity Experiment (NGE). A centrifugal pump was used to establish the liquid flow in a square $2.54 \times 2.54 \times 75-\mathrm{cm}$ flow channel which was constructed from acrylic material and pressure tested to $3.78 \times 10^{6} \mathrm{~Pa}(37.5$ psig) with no water leaks observed. The liquid flow rate was measured with one of the two liquid flow meters connected in parallel and used to accommodate the liquid flow rates that ranged from 0 to $6000 \mathrm{ml} / \mathrm{min}$. The gas flow rate was at first established from a pressurized air bottle. However, due to the need to establish low air flow rates, a motorized syringe pump was used. The latter established the desired-full g-calibrated rates up to $0.22 \mathrm{~cm}^{3} / \mathrm{s}$. A bypass loop was also established in order to have better control on the liquid flow rates. The liquid was pumped into the channel and its flow rate was controlled. The syringe pump was then activated and allowed to run until sufficient pressure was built in the syringe in order to prevent any liquid back-flow into the syringe. Then a gate valve was opened to allow the air into the liquid cross flow. Two orifice diameters $\left(D_{N}=0.033 \mathrm{~cm}\right.$ and $\left.0.076 \mathrm{~cm}\right)$ were used (one at a time) for air injection into the liquid flow. The orifices were flush with the surfaces in order to reduce flow disturbances. A high-speed camera (nac 500 or nac 1000) was used to videotape the events of bubble generation and detachment. Figure 2 shows a schematic of the flow diagram of the ground flow loop.

\subsection{Low-Gravity Two Phase Flow Loop}

This flow loop is frequently used for two-phase flow experiments requiring low gravity (McQuillen and Neumann, 1995). It consists of a liquid flow subsystem that is composed of a water reservoir made of acrylic material and fitted with a piston which is driven by air 
pressure provided by air bottle on board of the DC-9 platform. The loop has a gas flow subsystem that provides the gas flow into the liquid in order to establish the various regimes of two-phase flows. The Bubble Formation and Detachment Low Gravity Experiment (LGE) was performed using this loop. LGE concentrated on smaller orifices, lower gas and liquid flow rates. Since the existing two-phase flow loop gas flow system could not accommodate the required low gas flow rates for LGE, the same motorized syringe pump used in NGE was integrated into the loop in order to establish the gas flow rates. The LGE flow channel was the same as the one used for the NGE. The gas injection for LGE was established through three orifices, (of diameters $0.033,0.076$, and $0.15 \mathrm{~cm}$ ) which were positioned flush with the flat surface of the channel to generate air bubbles in the cross flow. Data acquisition was furnished by the two-phase flow loop as well. Liquid and gas flow rates as well as acceleration data were written to binary files and stored onto the loop on-board computer. Flow visualization was accomplished using the same high-speed cameras used in the normal$\mathrm{g}$ experiments. Figure 3 shows the overall test arrangement schematically for the reduced gravity experiment.

\section{EXPERIMENTAL PROCEDURE AND DATA ANALYSIS}

The procedure for LGE was similar to the one applied for NGE. The liquid flow was first established, then air was introduced through the orifice of choice. Several liquid flow rates were accomplished with several gas flow rates for each of the orifices. The recorded video data was analyzed using the TRACKER software (Klimek and Wright 1996). This enabled the measurement of the bubble diameter within $2-4 \times 10^{-3} \mathrm{~s}$ from the detachment 
point. The measurement was based on a count of pixels and a comparison with an existing scale. These measurements were entered in a text/data file that was read by a MATHEMATICA $\otimes^{\mathrm{TM}}$ program that calculated the bubble average diameter and the standard deviation of the mean. The low gravity period of $15-20 \mathrm{~s}$ per parabola was established by the DC-9 Low Gravity Platform flown from the NASA-Glenn Research Center at Lewis Field. The DC-9 flew several series of consecutive parabolas every mission. As the airplane begins climbing, the engine throttle is reduced to idle at the inflection point of the parabola. This action sets the payload in a free fall with the airplane that lasts between 15-20 s. As the airplane traverses the apex of the parabola and begins its descent, it begins accelerating and reaches an acceleration of $\sim 2 \mathrm{~g}$ at the bottom of the valley between parabolas. The distance between the apex and valley of traveled parabolas is on the order of 2,500 to $3,000 \mathrm{~m}$. The low gravity acceleration levels achieved by the DC-9 are between $0.01 \mathrm{~g}$ and $0.001 \mathrm{~g}$ during the low gravity periods.

\subsection{Experimental Uncertainties}

The experimental uncertainties are summarized as follows. The liquid flow rate in the channel was known to within $3 \%$ for the ground experiment and to within $5 \%$ on the twophase flow loop. The gas flow rate, provided by the syringe pump, was calibrated using two methods. The first involved timing the syringe pump as the piston traveled the syringe length and discharged bubbles into a cross flow of liquid. The other was to discharge the air in an inverted graduated cylinder filled with distilled water and to time the discharge process. Both methods used to calibrate the volumetric flow rate of air resulted in good agreement. The bubble diameter at detachment was measured, as previously mentioned, using the 
NASA-GRC developed TRACKER system. The error involved in the measurement was small $\approx 2.5-5 \%$ and this was due to the uncertainty in determining the boundary of the bubble as the diameter was measured.

\subsection{Average Gas Flow Rate during Bubble Formation and Detachment}

The average gas flow rate during bubble formation and detachment in low gravity as well as under full gravity was measured from the bubble diameter at detachment and the time elapsed from the beginning of bubble formation to the point of detachment. For the small orifice diameter of $0.033 \mathrm{~cm}$, the average flow rate differed much from the flow rate prescribed by the motor of the piston plunger. This difference was smaller for the intermediate orifice diameter of $0.076 \mathrm{~cm}$ and almost null for the largest orifice diameter of $0.15 \mathrm{~cm}$. The average gas flow rate $Q_{\mathrm{B}}$ ranged from 0.43 to $1.4 \mathrm{~cm}^{3} / \mathrm{s}$ for $D_{N}=0.033 \mathrm{~cm}$, whereas, for $D_{N}=0.15 \mathrm{~cm}, Q_{g}$ ranged from 0.018 to $0.22 \mathrm{~cm}^{3} / \mathrm{s}$ which is the range of the syringe pump. We report in this paper the average flow rate of gas that prevails during the event of bubble formation because of its relevance to the physical process of bubble evolution and detachment in cross flow. The difference between the prescribed flow rate by the syringe pump and the average flow rate measured from the bubble diameter and time to detachment is attributed to the interfacial pressure. As the orifice diameter is made smaller, the interfacial pressure, which varies as $4 \sigma / \mathrm{D}_{\mathrm{N}}$ ( $\sigma=$ surface tension), increase significantly. This results in time intervals when bubbles are not produced because the pressure inside the piston chamber is building up to overcome the interfacial pressure. When the pressure inside the piston becomes higher than the interfacial and liquid pressures, the gas is ejected from the orifice. When the total ejected mass is integrated over the pressure buildup and bubble 
formation times, it comes out to correspond to the prescribed flow rate. As the orifice diameter is increased, the interfacial tension pressure is decreased by the ratio of the orifice diameter. This reduces the pressure buildup time and makes the average flow rate very close to the prescribed flow rate.

\subsection{Liquid Velocity Profile at Injection Site}

The velocity profile in the test section was not measured. Instead, it was estimated using the theoretical work of Sparrow et al. (1964) for the hydrodynamic entrance region of pipes and ducts, which assumes that the initial profile is uniform and that steady state prevails. The derived velocity profile was used to evaluate the profile at the location of the various orifices along the channel length and to calculate the boundary layer thickness at these locations. Figure 4 shows the velocity profile at the location of the smaller orifice of diameter $0.033 \mathrm{~cm}$ which was at $30 \mathrm{~cm}$ from the channel entrance, and the larger orifice of diameter $0.15 \mathrm{~cm}$ which was at a distance $40 \mathrm{~cm}$ from the entrance. From the profiles shown in Fig. 4, one sees that the flow is almost fully developed at the location of the orifices with a slight decrease of the boundary layer thickness with increasing cross liquid velocity.

\section{RESULTS AND DISCUSSION}

\subsection{Important Forces}

The bubble diameter at detachment is dependent on the flow geometry, the flow conditions and the gravitational acceleration. Specifically, it is known (e.g. Kim et al. 1994) 
that the bubble diameter $\left(D_{B}\right)$ is affected mainly by the following quantities.

$$
D_{B}=f\left(D_{P}, D_{N}, U_{L}, Q_{g}, \rho_{L}, \rho_{g}, \mu_{L}, \mu_{g}, g\right)
$$

where $D_{P}$ is the test section hydraulic diameter, $U_{L}$ the superficial liquid velocity, $\rho$ the density, and $\mu$ the dynamic viscosity. The subscripts $\mathrm{L}$ and $\mathrm{g}$ are for the liquid and gas phase, respectively. In the present experiment, only $\mathrm{D}_{\mathrm{N}}, \mathrm{U}_{\mathrm{L}}$, and $\mathrm{Q}_{\mathrm{g}}$ are varied. Although the above relation can be non-dimensionalized using the Buckingham-PI theorem, it is important to note that the resultant dimensionless parameters are global parameters and they do not necessarily render the complete physical picture which describes the bubble generation. In order to describe the process in terms of proper dimensionless parameters it is necessary to have an accurate understanding of the physics involved in bubble formation. Therefore, the main objective of the present experimental work is to obtain a better understanding of fundamental physics of the whole process. For that reason, we will first deal with dimensional variables in the present work. Some dimensionless parameters will be introduced as needed in later discussion.

We interpret the present experimental data in terms of important forces involved in the process of bubble formation. The important forces in the present configurations are similar to those considered in a co-flow configuration (Bhunia et. al. 1998); buoyancy force, surface tension force, gas momentum, liquid drag, and liquid inertia. The buoyancy force,

$$
F_{B}=\pi / 6\left(\rho_{L}-\rho_{g}\right) D_{B}{ }^{3} g j
$$

acts vertically upward and it tends to detach the bubble from the orifice. On the other hand, 
the surface-tension force,

$$
\boldsymbol{F}_{\sigma}=-\pi \sigma D_{N} \boldsymbol{j}
$$

which acts along the rim of orifice, is an attaching force. The momentum flux associated with gas injection,

$$
F_{M}=\rho_{g} Q_{g}{ }^{2} /\left(\pi / 4 D_{N}{ }^{2}\right) j
$$

is a detaching force. The bubble experiences drag force exerted by the liquid flow. The liquid drag force acts both in the $\mathrm{x}$-direction (direction of liquid cross-flow) and in the $\mathrm{y}$ direction (direction of gas injection). Since the bubble is generally smaller than the channel hydraulic diameter in the present tests, the local liquid velocity $\left(U_{L}{ }^{*}\right)$ is smaller than $U_{L}$. Assuming that the liquid flow in the pipe has a parabolic velocity distribution approximately, one can relate $\mathrm{U}_{\mathrm{L}} *$ to $\mathrm{U}_{\mathrm{L}}$ as,

$$
U_{L}^{*}=2 U_{L}\left(I-\left(\left(D_{P}-D_{B}\right) / D_{P}\right)^{2}\right)
$$

Equation 5 calculates the local fluid velocity at the bubble center. The velocity of bubble center in the $\mathrm{x}$ direction is obtained by dividing the $\mathrm{x}$ location of bubble center at detachment by the total formation time. Using this average bubble velocity $\left(U_{B}\right)$, the liquid drag in the $x-$ direction can be expressed as

$$
F_{D x}=1 / 2 C_{D} \rho_{L}\left(U_{L}^{*}-U_{B}\right)^{2} \pi / 4 D_{B}^{2}
$$

where $C_{D}$ is the drag coefficient. The Reynolds number for the liquid flow around the bubble is given as $\operatorname{Re}_{B}=\rho_{L}\left(U_{L}^{*}-U_{B}\right) D_{B} / \mu_{L}$. As will be shown later, the bubble shape remains nearly 
spherical during its formation in the reduced gravity tests, the velocity of its center in the $y$ direction can be estimated as $V_{B}=1 / 2\left(d D_{B} / d t\right)$, where $t$ is time. From the conservation of mass, we have $\pi / 6 \mathrm{D}_{\mathrm{B}}{ }^{3}=\mathrm{Q}_{\mathrm{B}} \mathrm{t}$, so that $\mathrm{dD}_{\mathrm{B}} / \mathrm{dt}=\mathrm{Q}_{\mathrm{g}} /\left(\pi / 2 \mathrm{D}_{\mathrm{B}}{ }^{2}\right)$. Then, the liquid drag in the $\mathrm{y}$ direction can be estimated as,

$$
F_{D y}=1 / 2 C_{D} \rho_{L} V_{B}^{2} \pi / 4 D_{B}^{2}=\rho_{L} C_{D} Q_{g}^{2} /\left(8 \pi D_{B}{ }^{2}\right),
$$

with the associated Reynolds number based on $V_{B}$. The liquid drag in the $x$-direction is detaching, while that in the y-direction is attaching.

The drag coefficient $C_{D}$ is a function of Reynolds number. The bubble Reynolds number is less than 1000 in the present tests. As shown in our past modeling work (Kim et al. 1992; Bhunia et al. 1998), although the bubble has a free surface, the drag coefficient for a solid sphere tends to give a better agreement with experimental data. That is caused probably by the fact that the internal circulation of gas inside the bubble is disturbed by the injected gas momentum and also by the fact that the bubble free surface is partially immobilized by surface contamination. Thus, the drag coefficients used in the present analysis are (Bhunia et al. 1998);

$$
C_{D}=\left\{\begin{array}{l}
\frac{18.5}{\mathrm{Re}_{B}} \cdots 2 \leq \mathrm{Re}_{B} \leq 500 \\
0.44 \cdots 500 \leq \mathrm{Re}_{B} \leq 1000
\end{array}\right.
$$

The liquid inertia force is associated with the acceleration of liquid caused by the bubble motion and expansion, and acts both in the $\mathrm{x}$ and $\mathrm{y}$ directions. Using the aforementioned average bubble center velocity $U_{B}$, the relative velocity between the bubble and the liquid 
flow can be expressed as $U_{B}-U_{L}^{*}$. As will be shown later, the change of relative velocity during the bubble formation is generally small in the present tests. In that case, it can be shown that the liquid inertia force can be estimated as,

$$
F_{I x}=\rho_{L} C_{M C} Q_{g}\left(U_{B}-U_{L}^{*}\right)
$$

where $\mathrm{C}_{\mathrm{MC}}$ is the added mass coefficient. Since the relative velocity is always negative in the present tests, the liquid inertia in the $\mathrm{x}$-direction is detaching. The added mass coefficient $\mathrm{C}_{\mathrm{MC}}$ in the $\mathrm{x}$-direction is set equal to $19 / 32$ (Kim et al. 1994). Using the bubble center velocity $V_{B}$ in the $y$-direction given above, it can be shown that the liquid inertia in the $y$ direction is given as,

$$
F_{l y}=\rho_{L} C_{M C} Q_{g}^{2} /\left(3 \pi D_{B}^{2}\right) \text {, }
$$

and it is attaching. The value of $\mathrm{C}_{\mathrm{MC}}$ in the $\mathrm{y}$-direction used in the present analysis is $11 / 16$ (Kim et al. 1994).

In addition to the above forces, the bubble experiences a lift induced by the liquid shear flow in the present configuration. Based on the lift force associated with a sphere placed in a uniform shear flow (Auton, 1987), we compute the shear lift as,

$$
F_{S L}=\rho_{L} 1 / 2\left(\pi / 6 D_{B}^{3}\right)\left(U_{L}^{*}-U_{B}\right) d U_{L} / d y j
$$

The velocity gradient $d U_{V} /$ dy is estimated as $2 U_{L} / D_{P}$ in the present analysis.

Aboard the DC-9 aircraft the acceleration level and its direction change randomly during reduced gravity periods. However, the videotapes of bubble generation show that the bubble 
formation process was smooth in most cases. Although the g-jitter are partially responsible for the present data scatters (the repeatability of the measured bubble diameter at detachment is as large as $\pm 10 \%)$, it did not interfere with the fundamental process of bubble generation.

\subsection{Results from the NGE and LGE Experiments}

We first present the results of NGE and then lead into the LGE results. Figure 5 shows the bubble diameter at detachment plotted against the superficial liquid velocity for two different orifice diameters in normal gravity. When there is no liquid cross-flow, the important forces are buoyancy and surface tension. When the bubble diameter is small, the buoyancy force is small so that the surface tension keeps the bubble attached to the orifice (called expansion stage). When the buoyancy force becomes larger than the surface tension, the main bubble portion elevates from the orifice but is still connected to the orifice by a neck. The bubble keeps growing until the neck is pinched off (called detachment stage). The bubble diameter increases with $D_{N}$ as the attaching effects of surface tension increase. We observe in Fig. 5 that the liquid velocity has negligible effect, within experimental data scatter, on the bubble diameter in the present experiments. Therefore, buoyancy is still the main detaching force under the present conditions. We chose this condition because with much reduced buoyancy in low gravity one would expect a much different bubble formation process.

The bubble diameters measured in reduced gravity are given in Fig. 6. Generally, the bubble diameter in low-g is larger than that in normal gravity (Fig. 5) for a given nozzle diameter and gas volume flux. However, the difference seems to be smaller than what one would expect knowing that the bubble detaching force is much smaller in reduced gravity. 
The reason will be given later. Since the ratio of channel hydraulic diameter to orifice diameter is 2.8 or larger, the channel height does not constrain the bubble growth even for the largest bubble. As seen in Fig. 6 , the bubble diameter for $D_{N}=0.15 \mathrm{~cm}$ is affected appreciably by both $U_{L}$ and $Q_{B}$, but the bubble diameter for $D_{N}=0.033 \mathrm{~cm}$ is affected less by them. In fact, as will be discussed later, the bubble formation process for the tests with $D_{N}=0.033 \mathrm{~cm}$ and that for the tests with $D_{N}=0.15 \mathrm{~cm}$ are different, so the two cases are analyzed separately.

\subsubsection{Tests with $D_{N}=0.033 \mathrm{~cm}$-Dominant Gas Momentum Fluxe}

Figure 7 shows some typical traces of the gas-liquid interface of an evolving bubble in normal and reduced gravity for $D_{N}=0.033 \mathrm{~cm}$. In normal gravity (Fig. 7a), the bubble center moves mainly upward due to buoyancy. As can be seen in Fig. 7a, the bubble tends to spread along the wall in the beginning as it grows and thus its bottom is no longer anchored at the orifice. However, as the bubble increases its size, it rises quickly and the lower portion of the bubble becomes skinnier, forming a so-called neck. During this neck forming process, the contact line at the wall moves back toward the orifice. The neck is eventually pinched off while it is anchored at the orifice. Therefore, the attaching surface tension force is nearly equal to $\pi \sigma \mathrm{D}_{\mathrm{N}}$ at detachment. In reduced gravity (Fig. $7 \mathrm{~b}$ ), the bubble motion is still mainly upward (in the direction of gas injection). The bubble is pushed upward by the gas momentum, but its magnitude for the conditions of Fig. $7 \mathrm{~b}$ is about $5 \%$ compared to buoyancy in normal gravity. Consequently, the bubble in Fig. $7 \mathrm{~b}$ does not rise rapidly and no well-defined neck appears in reduced gravity. The bubble detaches simply as it is pulled away from the wall. One can then infer that the final bubble is formed after the expansion 
stage and there is no appreciable detachment stage in reduced gravity. This suggests that the final bubble size can be determined from the balance of attaching and detaching forces at the end of expansion stage. Generally, the bubble grows substantially in the detachment phase in normal gravity as additional gas is pumped into the bubble through the neck. For that reason, without the detachment phase in reduced gravity, the bubble does not become much larger than that in one-g, as observed in the present experiments. A detailed mechanism of bubble detachment will be discussed after analyzing the important forces in the process.

One important feature of the tests with $D_{\mathrm{N}}=0.033 \mathrm{~cm}$ is the relatively high gas flow rate. Then, it can be shown that the most important force is the one caused by the gas momentum. The gas momentum is the main detaching force in the y-direction. The ratio of liquid inertia to gas momentum in the $y$ direction ranges from 0.4 to 0.9 at detachment in the present tests but larger when the bubble is smaller. The expanding bubble also experiences an attaching liquid drag force in the negative y direction. Based on the expressions for various forces given earlier, the ratio of the liquid drag to the liquid inertia in the y-direction is given as $3 / 8\left(\mathrm{C}_{\mathrm{D}} / \mathrm{C}_{\mathrm{MC}}\right)$. The ratio is about 0.5 to 0.9 , so the liquid drag cannot be neglected. The liquid inertia and drag forces are both attaching, and decrease with increasing bubble size since they are inversely proportional to $D_{B}{ }^{2}$. In contrast, the gas momentum $\left(\rho_{B} Q_{B}{ }^{2} /\left(\pi / 4 D_{N}{ }^{2}\right)\right)$ remains constant during the bubble formation. Consequently, the bubble center is eventually lifted upward by the gas momentum. On the other hand, when the bubble is small, the liquid inertia and drag forces are relatively large, pushing the bubble towards the wall and causing the bubble to spread along the wall in the early stage of development, as seen in Fig. 7. The present data show that the average bubble center velocity in the $x$-direction, $U_{B}$, is nearly 
equal to the local liquid velocity $\left(U_{L}^{*}\right)$. This deems the shear lift force unimportant since the latter depends on the difference $\left(U_{L}^{*}-U_{B}\right)$.

The bubble is also pushed in the $\mathrm{x}$-direction by the combined force of liquid inertia and drag. Since the bubble is attached to the orifice, this combined force tilts the bubble-centeraxis in the downstream direction. However, the net displacement of the bubble center in the $\mathrm{x}$-direction during the whole bubble formation process is less than the bubble radius at detachment. In that situation, the bubble detachment from the orifice does not come from the force balance in the $\mathrm{x}$-direction, as will be discussed later.

As for the detachment mechanism, when the gas momentum becomes larger than the combined attaching force in the $y$-direction, the bubble center accelerates away from the wall and the liquid flows into the gap between the bubble bottom and the wall. During that process the liquid flow into the gap severs the connection between the bubble and the orifice, and bubble detachment eventually occurs when the liquid layer separates the bubble completely from the wall. Since the bubble axis is tilted, the final departure of the bubble from the wall occurs slightly downstream of the orifice. In contrast, if the bubble does not tilt, the bubble bottom would be eventually anchored at the orifice before detachment, which makes the bubble detachment more difficult than in the present configuration, as shown below.

Pamperin and Rath (1995) investigated bubble generation in a stagnant liquid under reduced gravity conditions. They showed that there exists a minimum gas flow rate below which no bubble detachment occurs. A Weber number based on the gas velocity and orifice 
diameter was formulated $\left(W e=\rho_{g} U_{g}^{2} D_{N} / \sigma\right)$, and it was shown that for We $<8$ the detachment did not occur in reduced gravity. Actually, the theoretical minimum value of We should be 4 , which is obtained by equating the gas momentum, $\rho_{g} \mathrm{Q}_{\mathrm{g}}{ }^{2} /\left(\pi / 4 \mathrm{D}_{\mathrm{N}}{ }^{2}\right)$, and the net surface tension force, $\pi \sigma D_{\mathrm{N}}$. In the present work with liquid cross-flow, the values of We (based on gas velocity) at detachment for the lowest liquid velocity $\left(\mathrm{U}_{\mathrm{L}}^{*}=1.4 \mathrm{~cm} / \mathrm{s}\right)$ ranges from 0.8 to 1.6 in the tests with $D_{N}=0.033 \mathrm{~cm}$. Clearly, the bubble detachment was made much easier, compared with the stagnant liquid configuration, even with the lowest liquid velocity in the present tests. The main difference is the bubble axis tilting with liquid cross-flow. Without tilting (no cross flow), the bubble is anchored at the orifice near detachment and the surface tension force becomes as large as $\pi \sigma D_{\mathrm{N}}$. On the other hand, when the bubble axis is tilted, the bubble is no longer attached to the orifice at detachment, as discussed above, so the net surface tension force in the y-direction is drastically reduced at detachment.

Based on the above discussion regarding the bubble detachment in reduced gravity with liquid cross-flow, one can say that the bubble detaches from the orifice when the combined attaching force (liquid inertia, liquid drag, and surface tension) becomes smaller than the detaching gas momentum. Then, if we neglect the surface tension force, one can determine the bubble size at detachment by equating the gas momentum and the combined liquid inertia and drag, which gives,

$$
\left(\frac{D_{B}}{D_{N}}\right)^{2}=\frac{1}{4} \frac{\rho_{L}}{\rho_{g}}\left(\frac{C_{M C}}{3}+\frac{C_{D}}{8}\right) .
$$

With $C_{M C}=11 / 16$ and $C_{D}$ ranging from 1.0 to 1.6 , Eq. (12) gives $D_{B} / D_{N}=9.4-10.4$ or $D_{B}=0.31$ - 
$0.34 \mathrm{~cm}$ for $D_{N}=0.033 \mathrm{~cm}$. The estimate is nearly independent of $Q_{g}\left(D_{B}\right.$ depends slightly on $\mathrm{Q}_{\mathrm{B}}$ through $\mathrm{C}_{\mathrm{D}}$ ). The measured bubble size is close to the estimate and does not depend much on $Q_{B}$ (Fig. 6). The result shows again that the surface tension force is substantially reduced in the present tests compared to bubble generation in a stagnant liquid or bubble generation in a cross-flow with gas injection through a nozzle. This reduction in surface tension force is an important aspect of the present work.

Figure 8 shows the bubble axis inclination angle at detachment, determined from the measured bubble center location at detachment. The inclination angle generally increases with increasing liquid velocity as the liquid inertia and drag in the $\mathrm{x}$-direction increases. The maximum inclination angle is about 40 to 50 degrees. Near the maximum angle, since the bubble shape is nearly spherical, the downstream displacement of the bubble center at detachment is nearly equal to the bubble radius. In that situation the bubble detaches from the orifice as the bubble bottom meniscus is pinched off, as in the larger orifice cases discussed below.

\subsubsection{Dominant Force Balance in Liquid Flow Direction}

\subsubsection{Tests with $\mathrm{D}_{\mathrm{N}}=0.15 \mathrm{~cm}$}

In the case of tests with $D_{N}=0.15 \mathrm{~cm}$, the gas flux is much smaller than that for the tests with $D_{N}=0.033 \mathrm{~cm}$. The gas momentum is less than $1 \%$ of that in the $D_{N}=0.033 \mathrm{~cm}$ tests but the liquid drag in the $x$ direction is substantially increased because of larger bubble size. Clearly, the force balance in the $\mathrm{x}$ direction is now more important in the bubble generation and, consequently, the details of detachment mechanism are expected to be different from the aforementioned mechanism for $D_{N}=0.033 \mathrm{~cm}$. The ratio of liquid inertia to drag in the $x$ 
direction is about $20 \%$ or smaller, so although the liquid drag is the most important detaching force, the liquid inertia cannot be neglected when $Q_{g}$ is large. The bubble center motion in the $\mathrm{x}$-direction is slow compared to the local liquid cross-flow velocity, so that the relative velocity in the liquid drag expression is taken to be just $U_{L}^{*}$ in the following analysis. Also, since the Weber number based on the local liquid velocity and the bubble radius, 0.4 or smaller, is larger than those in the tests with $D_{N}=0.033 \mathrm{~cm}$, the bubble shape is still nearly spherical but slightly more distorted.

The bubble diameters at detachment for various values of $Q_{g}$ and $U_{L}$ are presented in Fig. 9. The dependence of the bubble diameter at detachment on $\mathrm{Q}_{\mathrm{g}}$ and $\mathrm{U}_{\mathrm{L}}$ is also shown in Fig. 6. Unlike the result for $D_{N}=0.033 \mathrm{~cm}$, the bubble diameter is now a function of $Q_{B}$ and $U_{L}$. The fact that $D_{B}$ is strongly affected by $U_{L}$ suggests that the liquid drag is very important in the detachment, which is consistent with the above scaling analysis. Figure 9 shows that $D_{B}$ generally increases with increasing $Q_{g}$ and the effect of $Q_{g}$ increases with decreasing $U_{L}$. Those trends will be analyzed later.

Figure 10 depicts various bubble shapes during formation in reduced gravity. We define the bubble front side as the gas-liquid interface that is upstream of the bubble center. This makes the bubble backside as being the interface that is downstream of the bubble center of mass. We see from those traces that as the bubble grows, the bubble axis is tilted by the liquid flow. Then, when the bubble axis is tilted by a certain amount, the bubble bottom is pinched off starting from the front side and the bubble detaches from the orifice. The angle of inclination of the bubble axis at detachment is shown in Fig. 11 as a function of $U_{L}$ for various values of $\mathrm{Q}_{\mathrm{g}}$. The angle is nearly independent of $\mathrm{Q}_{\mathrm{g}}$ and increases slightly with 
increasing $U_{L}$. The result shows that the bubble detaches when the inclination angle becomes a certain value, about 50 degrees. As discussed earlier, with the inclination angle of about 45 degrees, the downstream displacement distance of the bubble center is nearly equal to the bubble radius, which means that the front side of bubble surface is nearly aligned with the front edge of the orifice. The sharp 90-degree edge of the orifice can anchor the bubble meniscus only up to the tangent angle of about 90 degrees from the wall. Consequently, the bubble cannot be anchored at the orifice if the tangent angle at the bubble front is larger than about 90 degrees and the bubble bottom is pinched off. In contrast, in the case of nozzle injection, the bubble is anchored at the nozzle tip so that the bubble meniscus can move around the tip more than it can around the orifice rim.

In terms of the force balance in the $\mathrm{x}$-direction, the combined liquid drag and inertia is the detaching force and the only attaching force is the surface tension force, so they must be balanced at detachment. For that reason, the ratio of the sum of the liquid drag and inertia to surface tension at detachment is plotted against $\mathrm{Q}_{\mathrm{g}}$ for three values of $\mathrm{U}_{\mathrm{L}}$ in Fig. 12. The data tend to scatter, however it is clear that the ratio does not depend much on the liquid velocity but is mainly a function of $\mathrm{Q}_{\mathrm{g}}$. The ratio generally increases with increasing $\mathrm{Q}_{\mathrm{g}}$, which is consistent with the trend of Fig. 9. It seems that the ratio becomes nearly constant, about 0.3 , when $\mathrm{Q}_{\mathrm{g}}$ is large. Kim et al. (1994) obtained the following expressions for the net surface tension force when the bubble axis is inclined:

$$
F_{\text {oxNet }}=\pi \sigma D_{N} f(\phi) \text {. }
$$

where 


$$
f(\phi)=\phi \operatorname{Cos} \phi\left((\pi / 2)^{2}-\phi^{2}\right)
$$

and $\phi$ is the bubble axis inclination angle from the local vertical (y-axis), and

$$
F_{\text {oyNet }}=\pi \sigma D_{N} g(\phi)
$$

where

$$
g(\phi)=\operatorname{Sin} \phi \phi
$$

According to Eqs. (13) and (14), the net surface tension force in the $x$-direction, when the bubble axis inclination angle is $45-50$ degrees, is $(0.3-0.33) \pi \sigma \mathrm{D}_{\mathrm{N}}$, which agrees well with the present result for large $Q_{g}$. The fact that the force ratio at detachment is less than 0.3 when $Q_{B}$ is smaller than about $0.1 \mathrm{~cm}^{3} / \mathrm{s}$ is analyzed below.

As for the force balance in the y-direction, the main force in the positive $y$-direction is the shear lift, which is opposed by the surface tension force. The surface tension force in the $y-$ direction at the time of detachment can be estimated based on Eq. (15). The forces computed based on the present data show that the ratio of the lift force to the surface tension force at detachment is about $35 \%$ or smaller, which means that the bubble detachment does not come from the force balance in the $y$-direction.

The fact that the detachment becomes easier with decreasing $Q_{\mathrm{g}}$ (Fig. 12) suggests that $\mathrm{Q}_{\mathrm{g}}$ affects the net surface tension force. Since the gas momentum is very small, the only possible way the gas flux affects the bubble formation is through the liquid inertia in the $\mathrm{x}$ direction. Although the liquid inertia is relatively small compared to the liquid drag at 
detachment as discussed above, it becomes more important in the earlier stage of development when $D_{B}$ is smaller, because while the liquid inertia does not depend on $D_{B}$, the liquid drag is proportional to $\mathrm{D}_{\mathrm{B}}{ }^{2}$. Consequently, during the time when the bubble is still small, the liquid inertia can become very important. In order to show that the trend of Fig. 9 can be explained by the liquid inertia, the data in Fig. 9 are plotted now against the ratio of liquid inertia to drag at detachment in Fig. 13. Also, the surface tension force is modified by factor $f(\phi)$ based on Eq. (13) in the figure. Figure 13 shows that the data can indeed be correlated by the ratio and that the net detaching force is reduced when the ratio is smaller than about 0.1 . Although it is difficult to observe the bubble meniscus shape close to the orifice cleanly, the backside of the bubble seems to lose the orifice pinning edge in an early stage of development when $\mathrm{Q}_{\mathrm{g}}$ is small. This loss of pinning is responsible for the reduction in the surface tension force.

The fact that the loss of pinning occurs when the liquid inertia is small can be explained as follows. Since the shear lift is proportional to $\mathrm{D}_{\mathrm{B}}{ }^{3}$, it is relatively small when the bubble is small. But, in the later stage it becomes important so that the bubble is pulled away from the wall by the shear lift, which helps to avoid the loss of pinning. Therefore, when the loss of pinning occurs, it happens in an early stage of development when the shear lift force is small, as observed experimentally. In the beginning the bubble meniscus is attached to the orifice, and then as the bubble axis tilts, the liquid in the back of the bubble is pushed away. Since the liquid inertia is associated with the acceleration of the surrounding liquid, it becomes more difficult (or requires larger force) to remove the liquid from behind the bubble, when the liquid inertia increases. Therefore, with increasing liquid inertia, the loss of pinning is 
delayed. When the ratio of liquid inertia to drag is larger than about 0.1 , the loss of pinning does not occur until detachment. When the pinning edge is lost in an early stage, the attaching surface tension force is reduced. As a result, the bubble size is still small when it detaches, so the shear lift does not restore the pinning.

\subsubsection{Tests with $\mathrm{D}_{\mathrm{N}}=0.076 \mathrm{~cm}$}

For the tests with $D_{N}=0.076 \mathrm{~cm}$, the gas momentum is much smaller than the forces in the $\mathbf{x}$ direction, so the forces in the $\mathbf{x}$ direction are mainly responsible for bubble detachment as in the tests with $D_{N}=0.15 \mathrm{~cm}$. However, unlike in the tests with $D_{N}=0.15 \mathrm{~cm}$, the liquid inertia force is generally comparable with the liquid drag in the $x$ direction. Since the bubble center velocity is $10-20 \%$ of $U_{L}^{*}$, the relative velocity is used in estimating the liquid drag and inertia.

The bubble axis inclination angle at detachment is shown in Fig. 14. The inclination angle is $30-40$ degrees, increasing slightly with the liquid velocity. The inclination angle at detachment is generally smaller than that in the tests with $D_{N}=0.15 \mathrm{~cm}$. The front side of bubble surface makes a nearly 90 -degree angle at that inclination angle and the bubble bottom is pinched off beyond that. As in the $\mathrm{D}_{\mathrm{N}}=0.15 \mathrm{~cm}$ tests, the ratio of combined liquid drag and inertia force to surface tension force is important for the bubble detachment. According to Eq. (13), the net surface tension force is reduced by factor $f(\phi)$ when the bubble axis is inclined. Therefore, the ratio of the combined detaching force to the reduced surface tension force is given in Fig. 15. As in Fig. 12, no systematic effect of $U_{L}$ is detected, so only the effect of $\mathrm{Q}_{\mathrm{g}}$ is emphasized in Fig. 15. The effect of $\mathrm{Q}_{\mathrm{g}}$ is represented by the ratio of liquid inertia to drag at detachment as discussed earlier. Figure 15 shows that the ratio of 
detaching and attaching forces is nearly unity, which means that the bubble bottom is supported at the orifice up to inclination angle $\phi$. The ratio of liquid inertia to drag is about unity in Fig. 15, so $Q_{g}$ does not affect the force balance within the experimental error.

From the tests with $D_{\mathrm{N}}=0.076$ and $0.15 \mathrm{~cm}$, one can conclude that the bubble detachment occurs when the bubble inclination angle becomes a certain value or when the front side of bubble surface becomes nearly normal to the wall, at which point the bubble bottom is pinched off. The inclination angle at detachment depends on the bubble meniscus shape near the orifice, which is a function of various parameters: ratio of bubble diameter to orifice diameter, the Weber number $\left(\rho_{\mathrm{L}} \mathrm{U}_{\mathrm{L}}{ }^{* 2} \mathrm{D}_{\mathrm{B}} / \sigma\right)$, the contact angle at the wall, and the liquid flow field around the bubble. Therefore, to predict exactly at what inclination angle the bubble detaches for given conditions is a very complex problem and beyond the scope of the present force balance analysis.

\section{CONCLUSION}

In the present work, we have studied bubble formation and detachment from an orifice positioned flush to the surface of a wall. We have investigated this problem with a liquid cross-flow perpendicular to the bubble injection direction and under normal and low gravity conditions, where the latter were furnished by the NASA DC-9 Low gravity platform. In normal gravity conditions, we found that the bubble formation and detachment follows the two-stage mechanism where the bubble forms during the expansion phase and lifts up and develops a neck during the detachment phase, and then the neck collapses and the bubble

departs. In the present tests the cross liquid velocity was found to have only a slight effect on 
the bubble diameter at detachment because of the dominant effect of buoyancy. Available detachment criteria in the cross-flow configuration are based on experimental information obtained in normal gravity.

Under low gravity conditions and in the present wall injection configuration, the mechanism of bubble formation and detachment changes significantly. The detachment phase observed under normal gravity is not present under low gravity, since a well-defined bubble neck does not form. Two different detachment mechanisms are identified in the present work. We have found that for the case of greater gas momentum flux force or higher gas flow rates $\left(\mathrm{D}_{\mathrm{N}}=0.033 \mathrm{~cm}\right)$, the bubble detachment is governed by balancing the combined force of liquid inertia and drag in the gas injection direction with the gas momentum flux. The surface tension force is much reduced because a large part of the bubble pinning edge at the orifice is lost as the bubble axis is tilted by the liquid flow. However, for lower momentum force or lower gas flow rates $\left(D_{\mathrm{N}}=0.15\right.$ and $\left.0.076 \mathrm{~cm}\right)$ when the bubble axis inclination angle exceeds a certain value or when the front side of bubble surface becomes nearly normal to the wall, the bubble bottom is pinched off starting from the front side and the bubble detaches from the orifice. The present work shows that in order to obtain detachment, one does not have to resort to high gas flow rate as in the experiment by Pamperin and Rath, (1995). One can inject bubbles with very small gas flow rates and use slow liquid cross velocity to help detach the evolving bubbles. As previously mentioned, understanding bubble formation and detachment is crucial to the understanding of two-phase flows which have several applications in space systems. 


\section{ACKNOWLEDGMENTS}

The work performed at Case Western Reserve University is supported by NASA under Grant NAG3-1913. 


\title{
NOTATION
}

\author{
$\mathrm{C}_{\mathrm{D}} \quad$ Drag coefficient \\ $\mathrm{C}_{\mathrm{MC}}$ Added mass coefficient \\ $\mathrm{D}_{\mathrm{B}} \quad$ Bubble diameter $(\mathrm{cm})$ \\ $D_{\mathrm{N}} \quad$ Diameter of injection orifice $(\mathrm{cm})$ \\ $D_{p} \quad$ Channel hydraulic diameter or pipe diameter $(\mathrm{cm})$ \\ $F_{B} \quad$ Buoyancy force (dynes) \\ $F_{D} \quad$ Dragforce (dynes) \\ $F_{I} \quad$ Inertia force (dynes) \\ $\mathrm{F}_{\mathrm{SL}} \quad$ Shear lift force (dynes) \\ $F_{M} \quad$ Momentum flux force (dynes) \\ $F_{\sigma} \quad$ Surface tension force (dynes) \\ g Gravitational acceleration $\left(\mathrm{cm} / \mathrm{s}^{2}\right)$ \\ j Unit vector in the y direction \\ $\mathrm{Q}_{\mathrm{g}} \quad$ Gas, dispersed phase-air flow rate $\left(\mathrm{cm}^{3} / \mathrm{s}\right)$ \\ $\mathrm{Re}_{\mathrm{B}} \quad$ Reynolds number based on bubble diameter \\ $\mathrm{Re}_{\mathrm{P}} \quad$ Reynolds number based on pipe/channel diameter \\ $\mathrm{t} \quad$ Time (s) \\ $\mathrm{U}_{\mathrm{B}} \quad$ Bubble velocity $(\mathrm{cm} / \mathrm{s})$ \\ Ug Gas velocity $(\mathrm{cm} / \mathrm{s})$ \\ $\mathrm{U}_{\mathrm{L}}{ }^{*} \quad$ Local superficial liquid velocity $(\mathrm{cm} / \mathrm{s})$ \\ $V_{B} \quad$ Bubble velocity component in y-direction $(\mathrm{cm} / \mathrm{s})$ \\ $\mathrm{U}_{\mathrm{L}} \quad$ Average superficial liquid velocity $(\mathrm{cm} / \mathrm{s})$ \\ We Weber number \\ $\mathrm{x} \quad$ Component of motion along the $x$ axis $(\mathrm{cm})$ \\ $y \quad$ Component of motion along the $y$ axis $(\mathrm{cm})$
}

\section{Subscripts}

B Bubble

g Disperse phase-Gas

L Continuous phase-Liquid

$\mathrm{N} \quad$ Orifice

$\mathrm{p} \quad$ Channel or pipe

$\mathrm{x}, \mathrm{y} \quad$ Rectangular coordinates

\section{Greek Symbols}

$\pi \quad$ Constant

$\phi \quad$ Tilt angle

$\rho \quad \operatorname{Density}\left(\mathrm{g} / \mathrm{cm}^{3}\right)$

$\sigma \quad$ Surface Tension Coefficient (dynes/cm)

$\mu \quad$ Dynamic viscosity $(\mathrm{g} / \mathrm{cm} s)$ 


\section{REFERENCES}

Al-Hayes, R. A. M. and Winterton, R. H. S. (1981) Bubble diameter on detachment in flowing liquids. Int. J. Heat Mass Transfer 24, 223-230.

Auton, T.R. (1987) The lift force on a spherical body in a rotational flow. J. FluidMech. 183, pp. 199-218.

Bhunia, A., Pais, S., Kamotani, Y., and Kim, I. (1998) Bubble formation in a coflow configuration in normal and reduced gravity. AIChE Journal 44, 1499-1507.

Eastman, R. E., Feldamis, C. J., Haskin, W. L., and Weaver, K. L. (1984) Two-phase fluid transport for spacecraft. AFWAL-TR-84-3028.

Kawase, Y. and Ulbrecht, J. J. (1981) Formation of drops and bubbles in flowing liquids. Ind. Eng. Chem. Process Des. Dev. 20, 636-640.

Kim, I., Kamotani, Y., and Ostrach, S. (1992) Modeling of bubble and drop formation in flowing liquids in terrestrial and microgravity environments. Report EMAE/TR-92-207, Department of Mechanical and Aerospace Engineering, Case Western University, Cleveland, $\mathrm{OH}$.

Kim, I., Kamotani, Y., and Ostrach, S. (1994) Modeling of bubble and drop formation in flowing liquids in microgravity. AIChE Journal 40, 19-28.

Klimek, R. B., Wright, T. W., and Sielken R. S. (1996) Color image processing and object tracking system. NASA TM-107144.

Kumar, R. and Kuloor, N. R. (1970) The formation of bubbles and drops. Advances in Chemical Engineering 8, 256-365.

Marshall, S.H. and Chudacek, M.W. (1993) A model for bubble formation from an orifice with liquid cross flow. Chem. Engn. Science 11, 2049-2059.

McQuillen, J. B., and Neumann, E. S. (1995) Two-phase flow research using the Learjet apparatus. NASA TM-106814.

Ostrach S. (1988) Industrial processes influenced by gravity. NASA CR-182140, C-21066G.

Pamperin, O. and Rath, H. (1995) Influence of buoyancy on bubble formation at submerged orifices. Chem. Engng. Sci. 50, 3009-3024.

Sparrow, E. M., Lin, S. H., and Lundgren, T. S. (1964) Flow development in the hydrodynamic entrance region of tubes and ducts. Phys. Fluids 7, 338-347. 
Tsuge, H., Hibino, S., and Nojima, U. (1981) Volume of a bubble formed at a single submerged orifice in a flowing liquid. International Chemical Engineering 21, 630-636.

Tsuge, H., Tanaka, Y., Terasaka, K., and Matsue, H. (1997) Bubble formation in flowing liquid under reduced gravity. Chem. Engng. Science 52, 3671-3676.

Wraith, A. E. (1971) Two-stage bubble growth at a submerged plate orifice. Chem. Engng. Sci. 26, 1656-1671. 


\section{FIGURE CAPTIONS}

Figure 1 Illustration of wall and nozzle bubble injection configurations.

Figure 2 Normal Gravity Experiment (NGE) flow loop.

Figure 3 Schematic of experimental setup for reduced gravity test.

Figure 4 Calculated velocity profiles across test section at orifice locations for (a) $\mathrm{D}_{\mathrm{N}}=0.15 \mathrm{~cm}$, and (b) $\mathrm{D}_{\mathrm{N}}=0.033 \mathrm{~cm}$.

Figure 5 Bubble diameter at detachment as a function of superficial liquid velocity in normal gravity.

Figure 6 Bubble diameter at detachment as a function of superficial liquid velocity in reduced gravity.

Figure 7 Traces of gas-liquid interface of evolving bubble for $D_{N}=0.033 \mathrm{~cm}$ in (a) normal gravity, and (b) reduced gravity.

Figure 8 Bubble axis inclination angle at detachment as a function of superficial liquid velocity for $\mathrm{D}_{\mathrm{N}}=0.033 \mathrm{~cm}$.

Figure 9 Bubble diameter at detachment as a function of gas flow rate for $D_{N}=0.15$ $\mathrm{cm}$.

Figure 10 Traces of gas-liquid interface of evolving bubble for $D_{N}=0.15 \mathrm{~cm}$ in reduced gravity.

Figure 11 Bubble axis inclination angle at detachment as a function of superficial liquid velocity for $D_{N}=0.15 \mathrm{~cm}, Q g=0.22 \mathrm{~cm}^{3} / \mathrm{s}$, and $U_{L}=7.7 \mathrm{~cm} / \mathrm{s}$.

Figure 12 Ratio of detaching force to surface tension force at detachment as a function of gas flow rate for $D_{N}=0.15 \mathrm{~cm}$.

Figure 13 Ratio of detaching force to surface tension force as a function of liquid inertia to drag ratio at detachment for $\mathrm{D}_{\mathrm{N}}=0.15 \mathrm{~cm}$.

Figure 14 Bubble axis inclination angle at detachment as a function of superficial liquid velocity for $D_{\mathrm{N}}=0.076 \mathrm{~cm}$.

Figure 15 Ratio of detaching force to surface tension force as a function of liquid inertia to drag ratio at detachment for $\mathrm{D}_{\mathrm{N}}=0.076 \mathrm{~cm}$. 
Wall bubble Injection

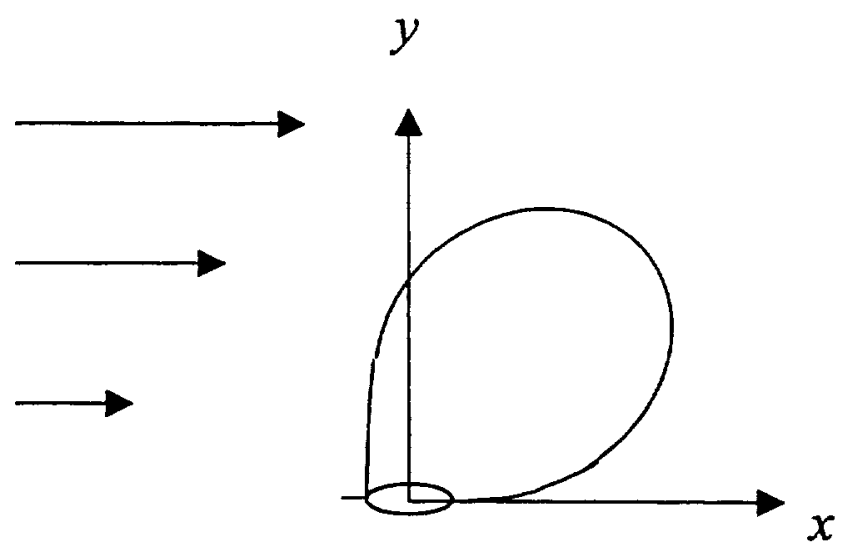

Nozzle Bubble Injection

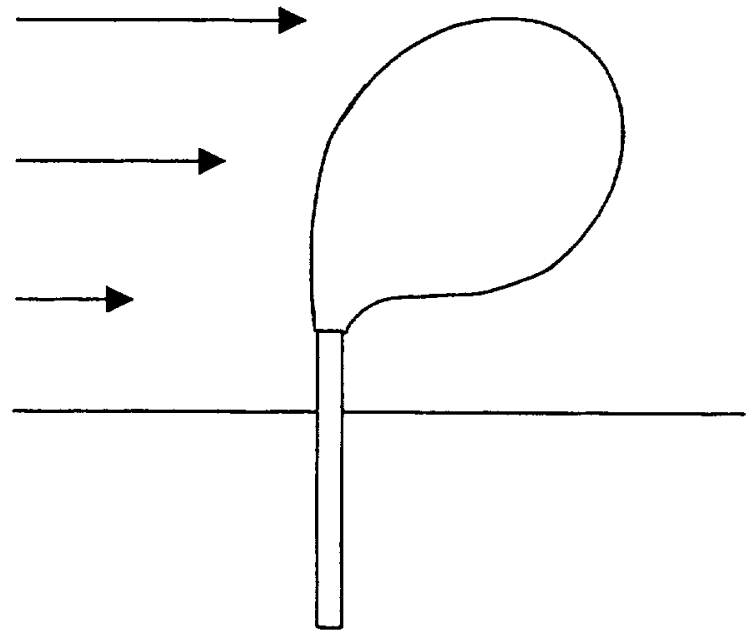

Fig 1 


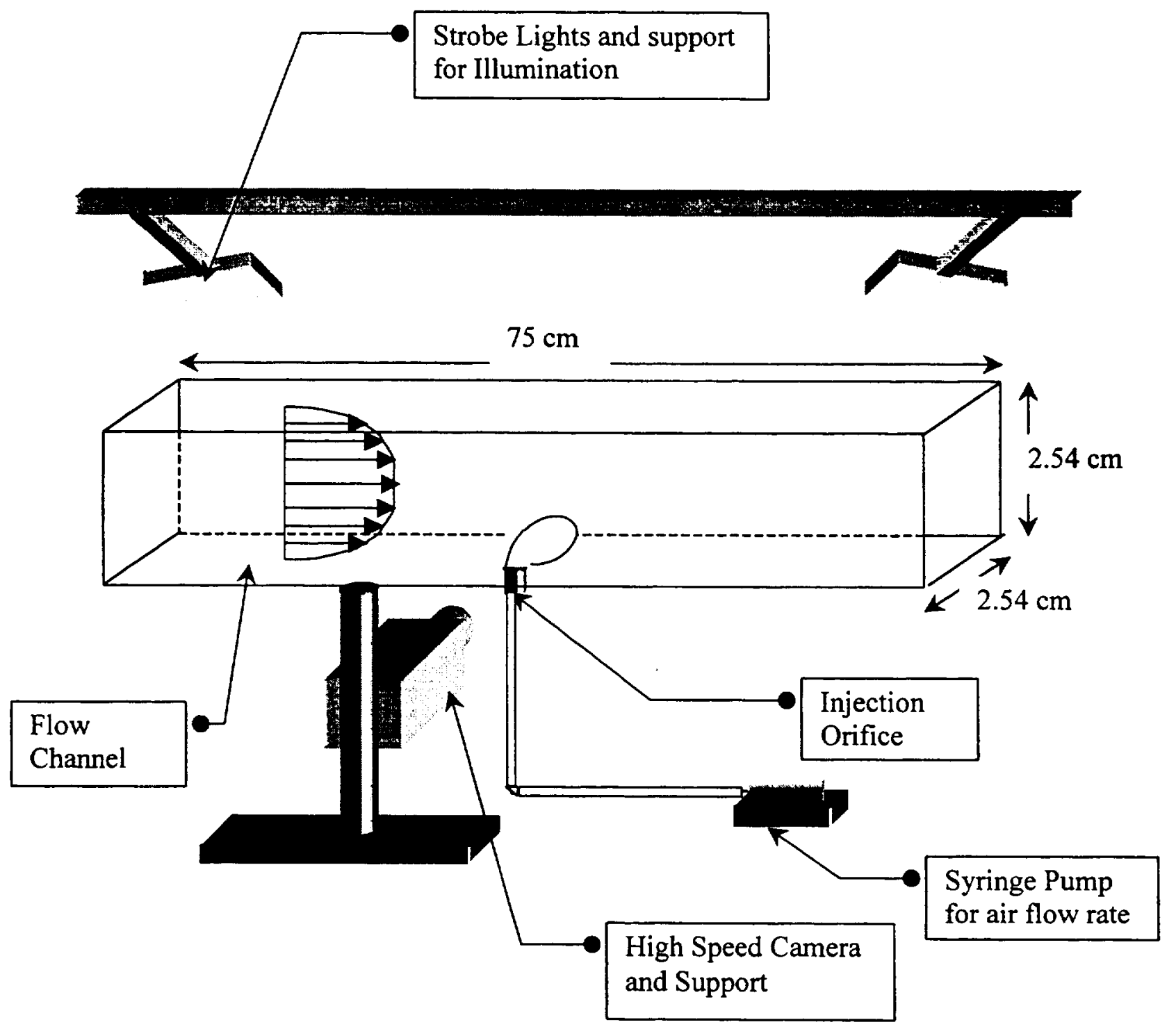

Fig. 3 


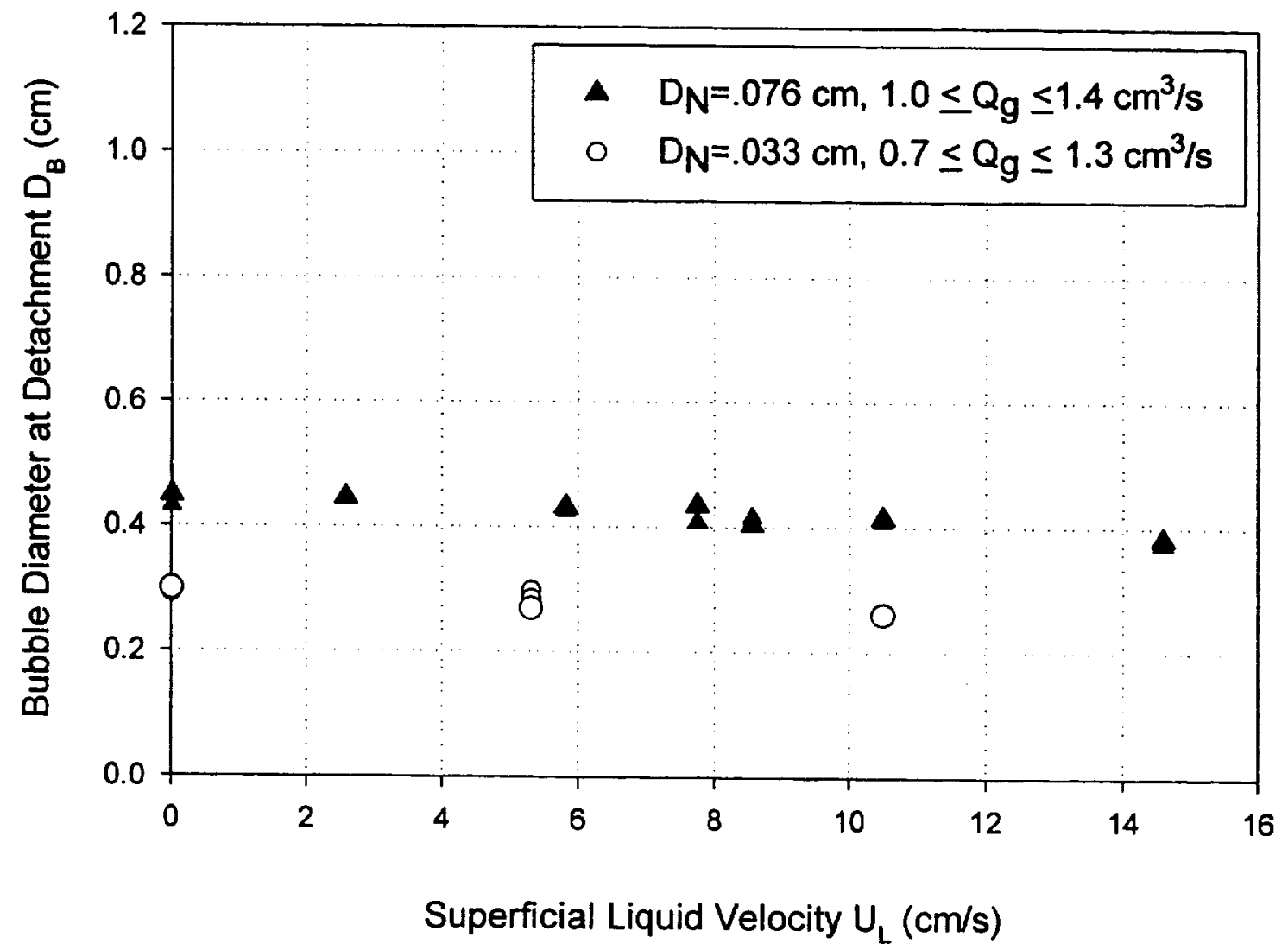

Fig. 5 


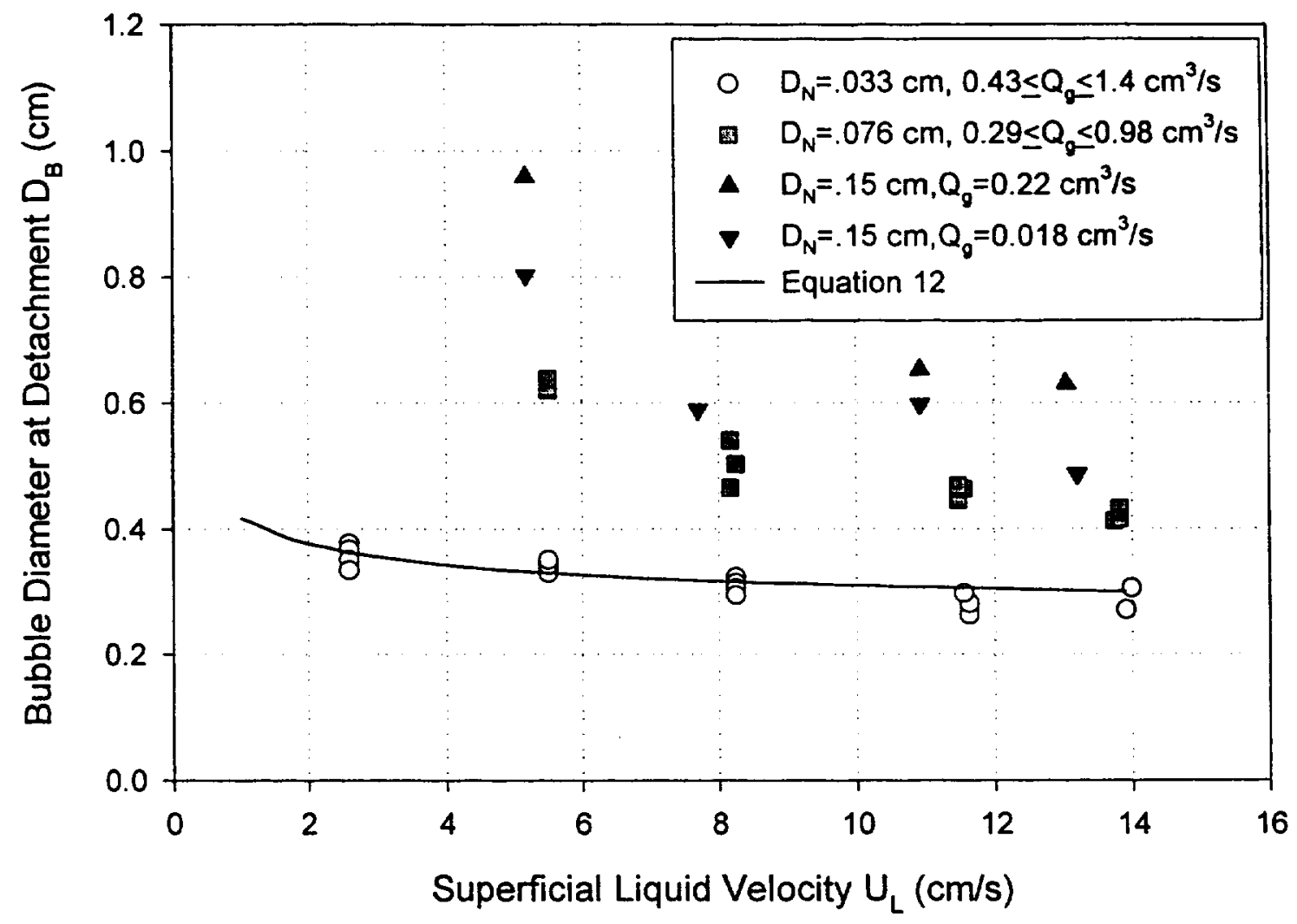

Fig. 6 


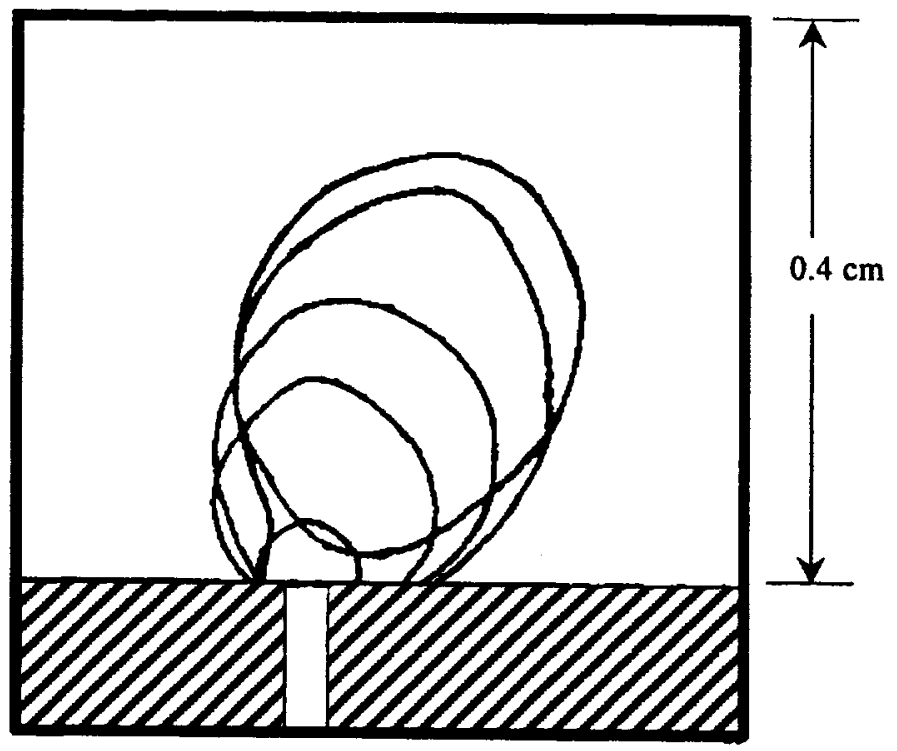

a)

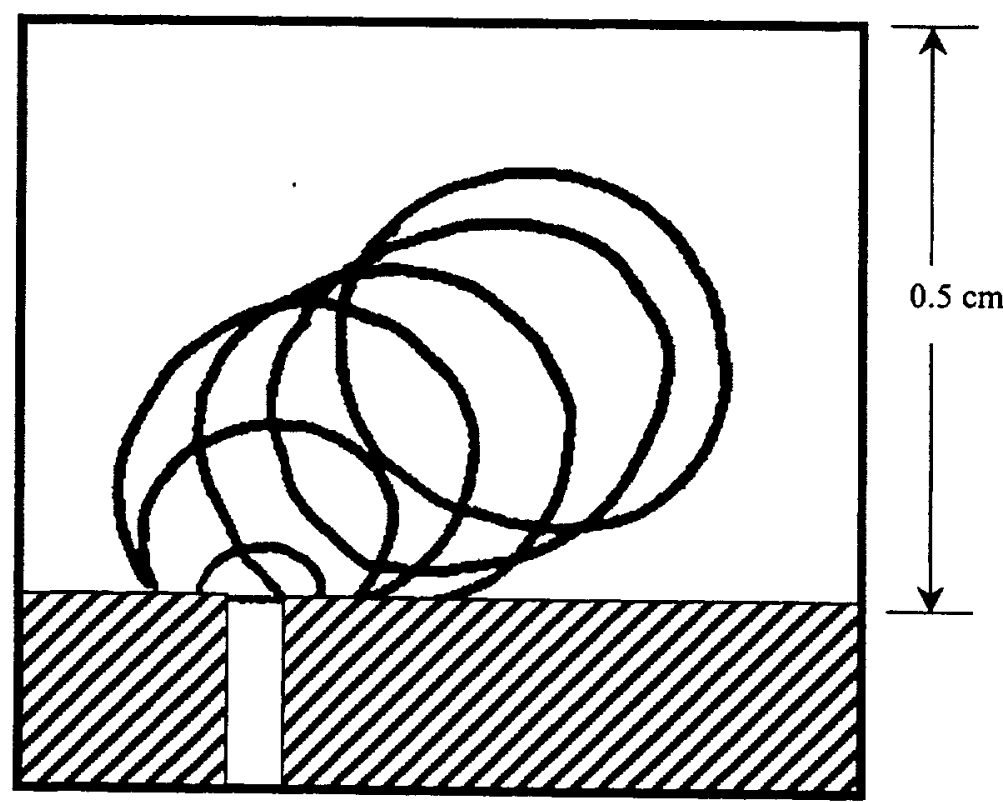

b)

Fig. 7 


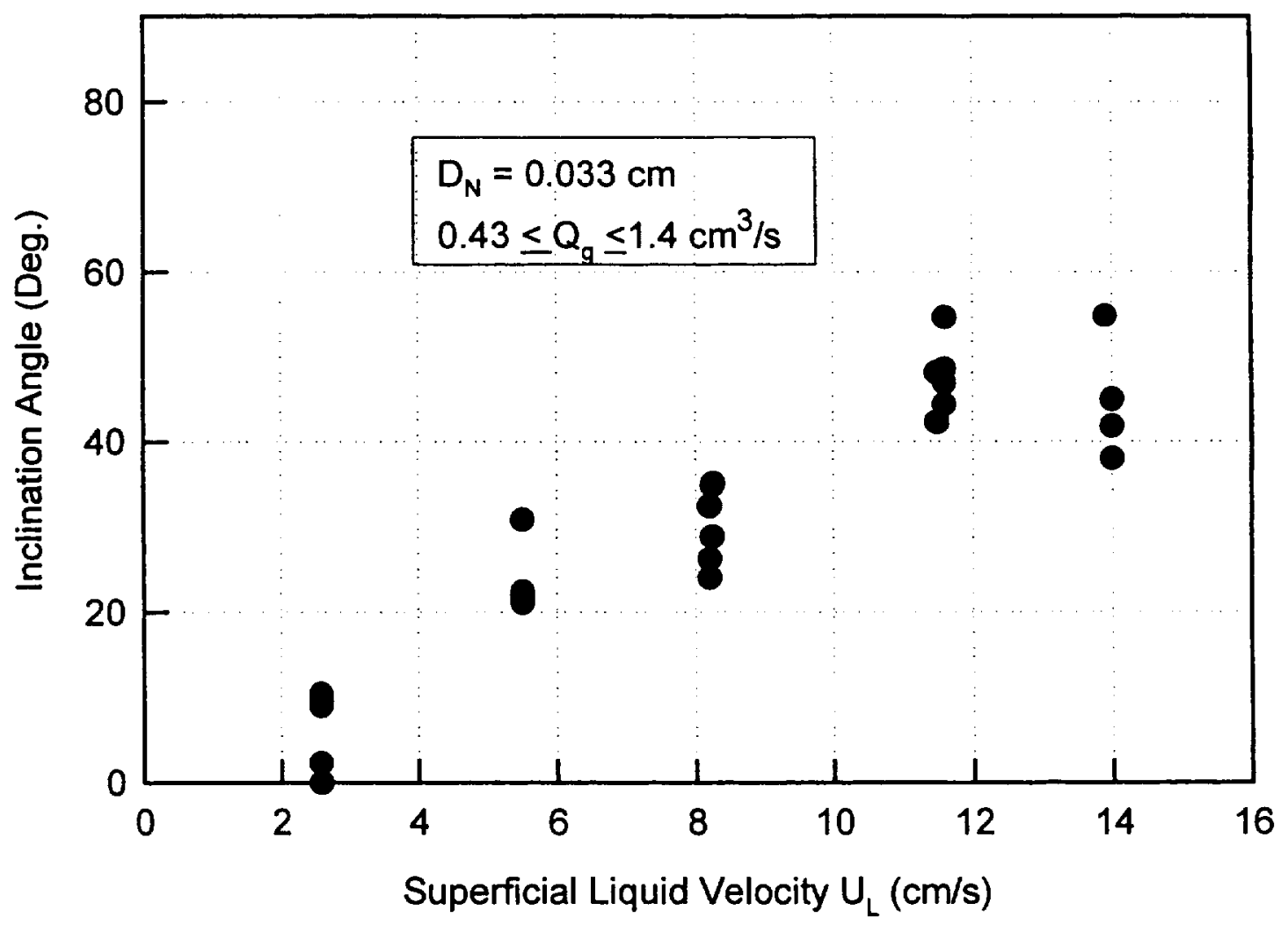

Fig. 8 


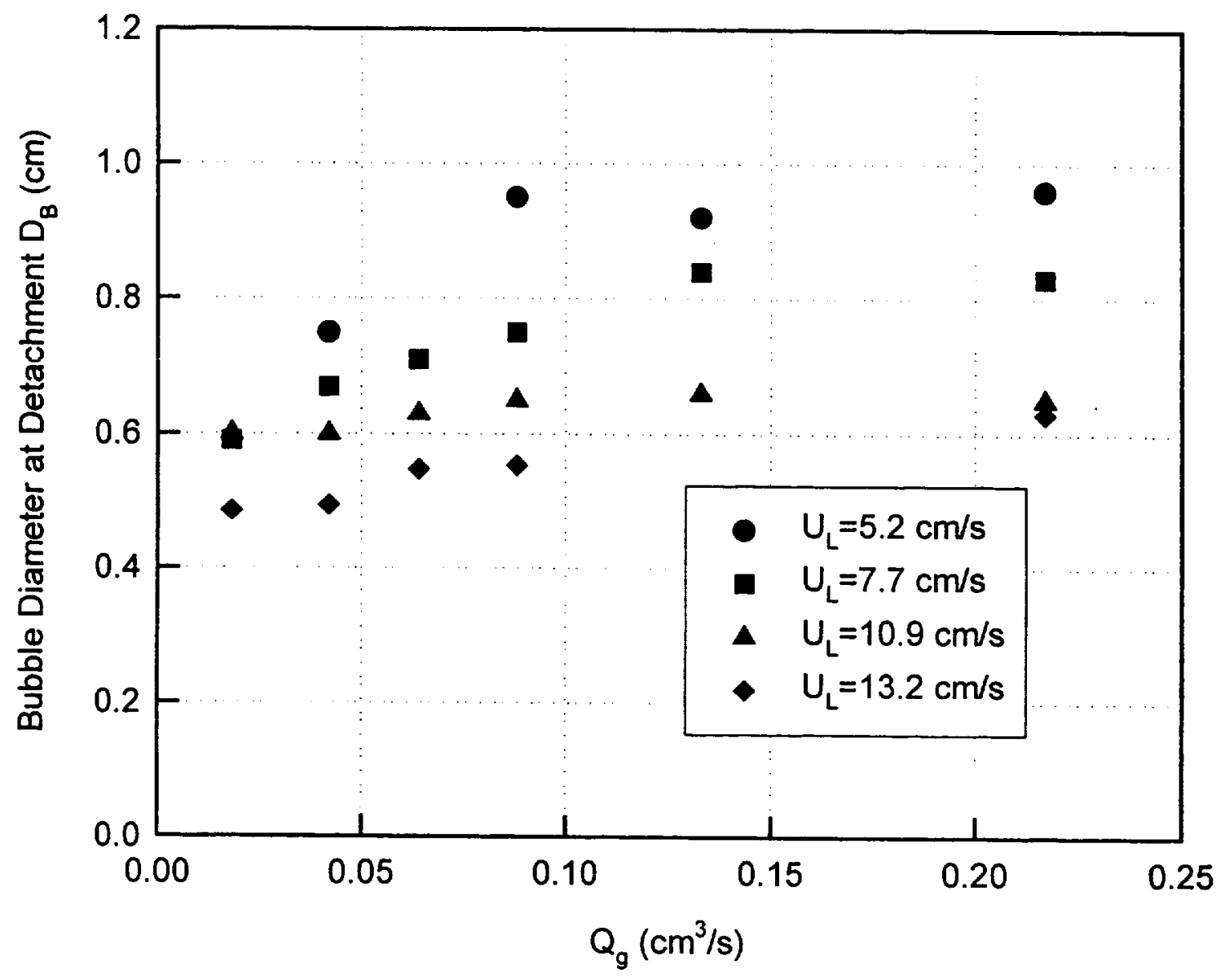

Fig. 9 


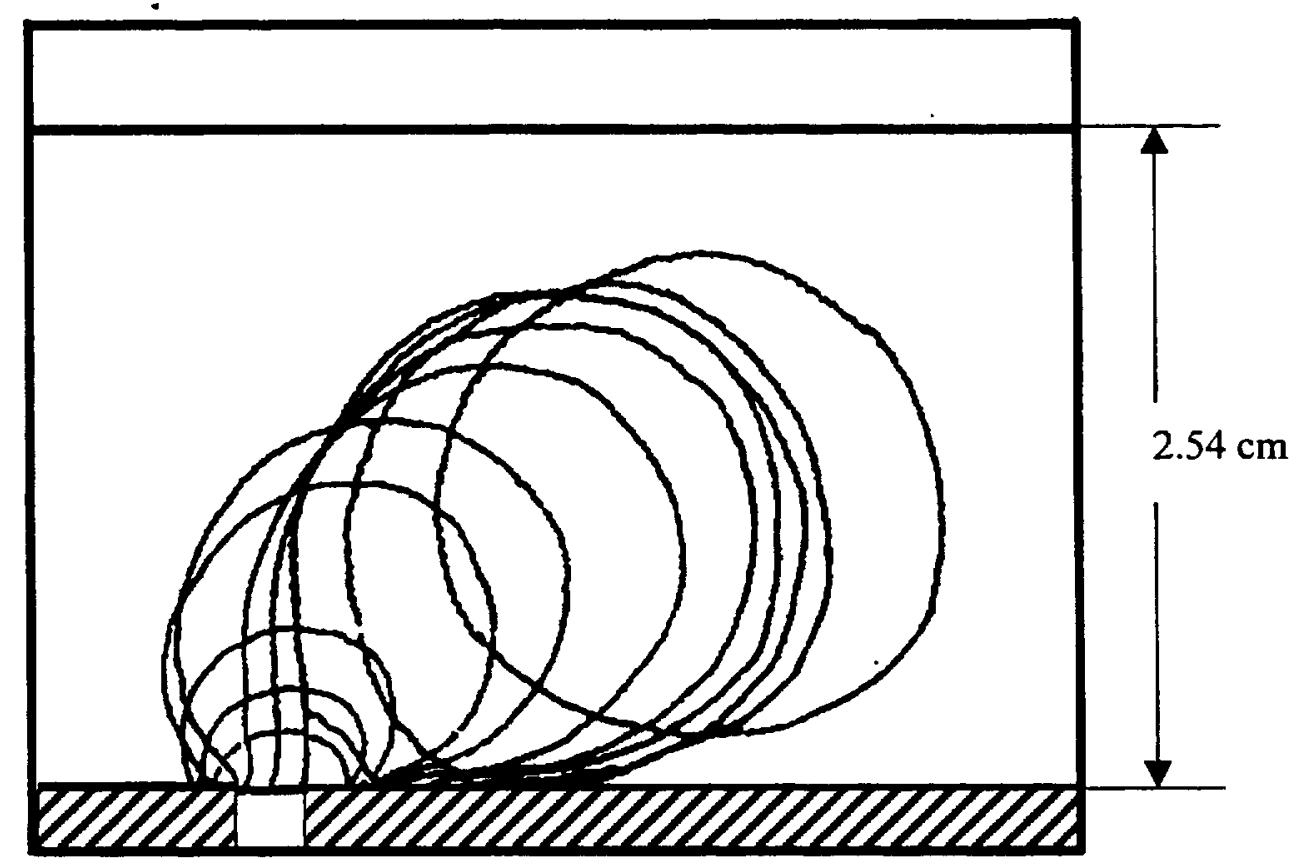

Fig. 10 


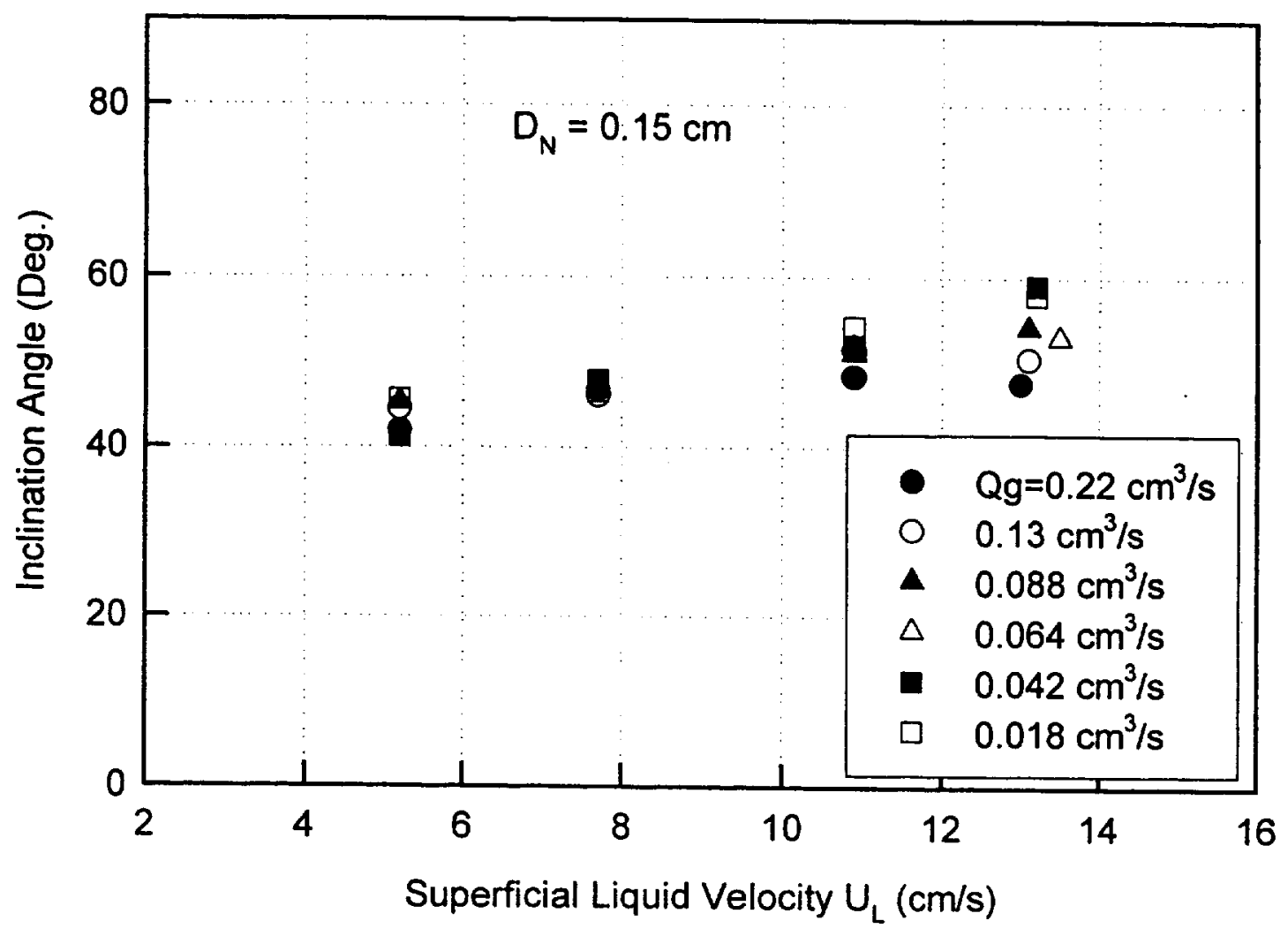

$F i g .11$ 


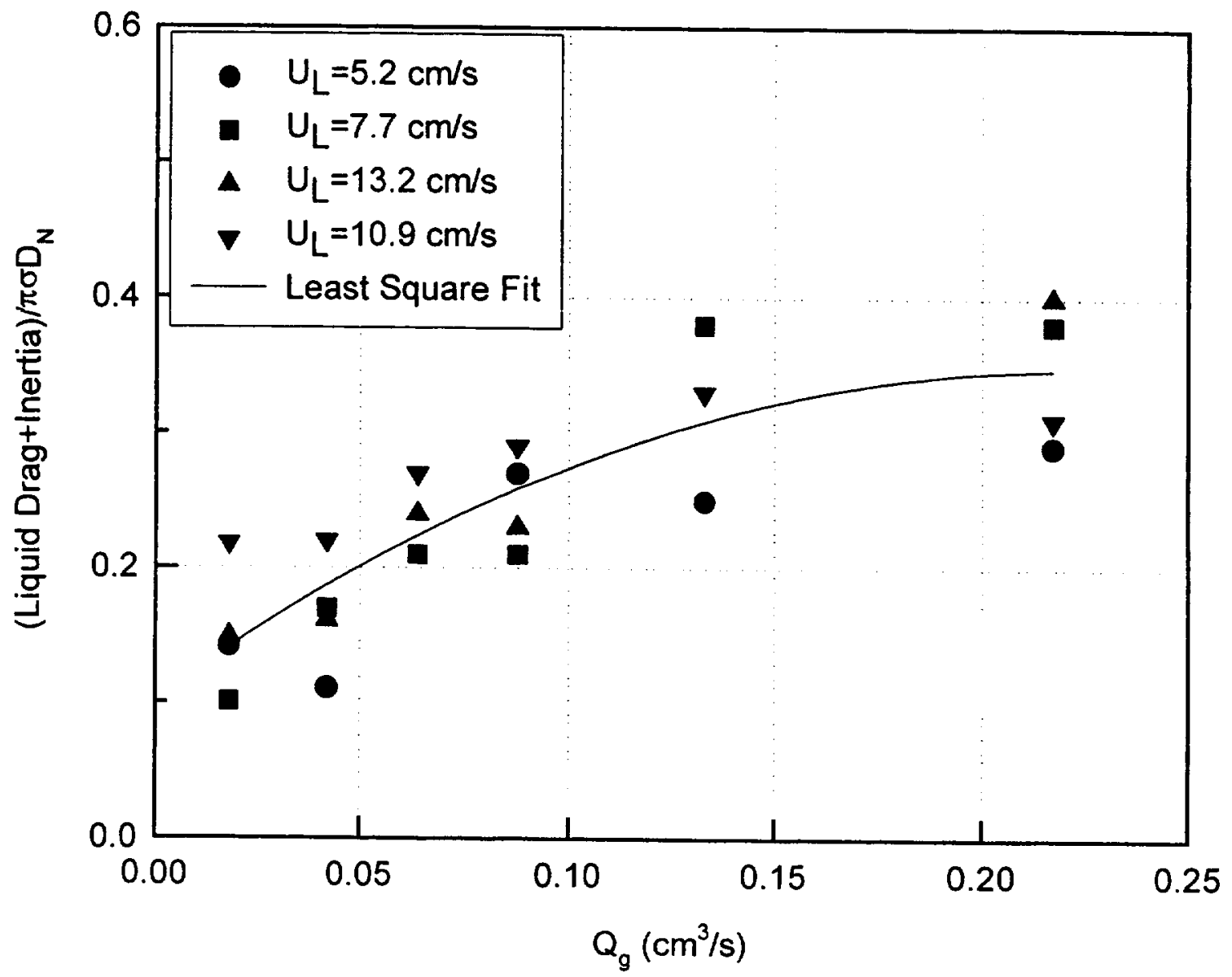

Fig. 12 


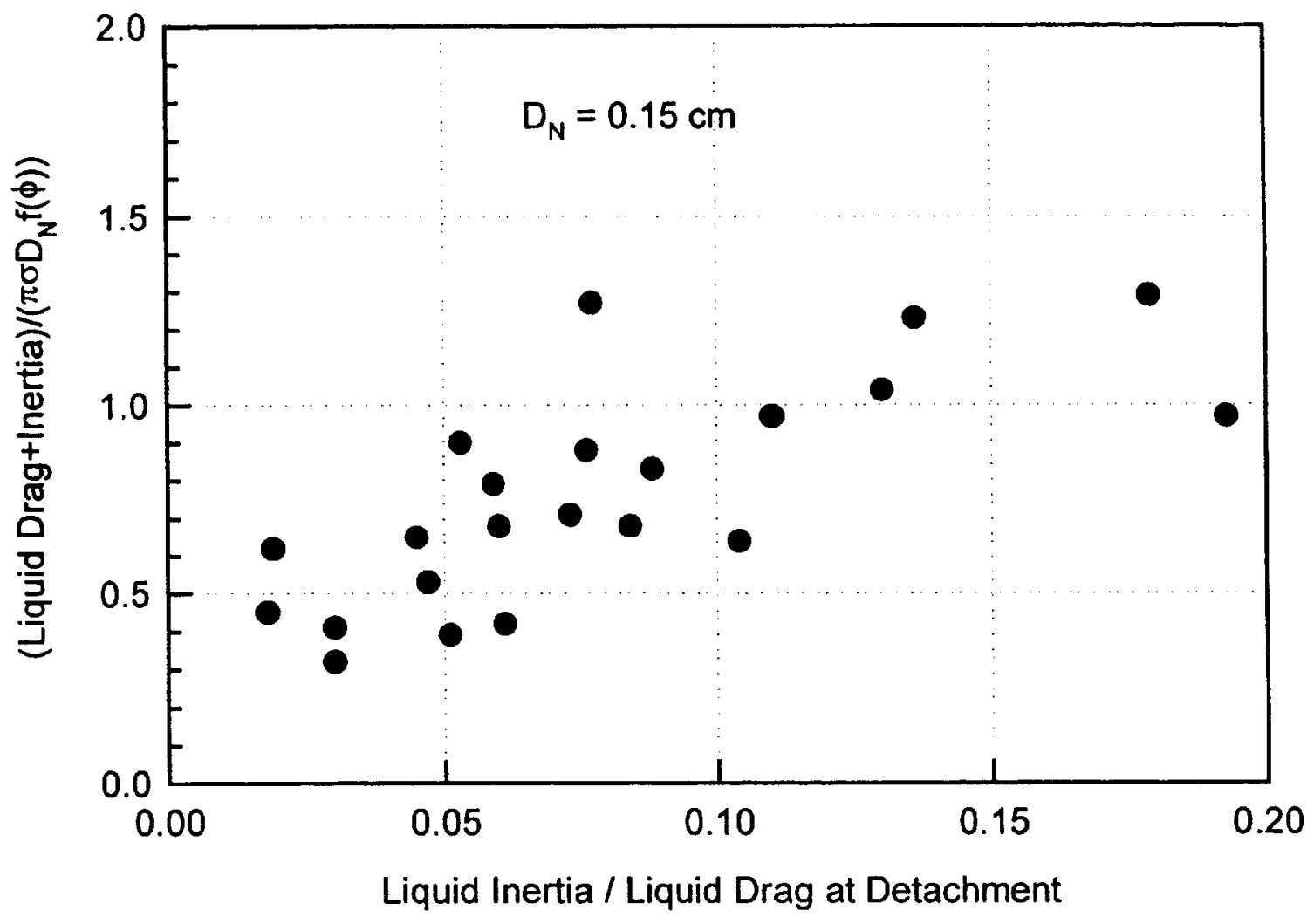

Fig. 13 


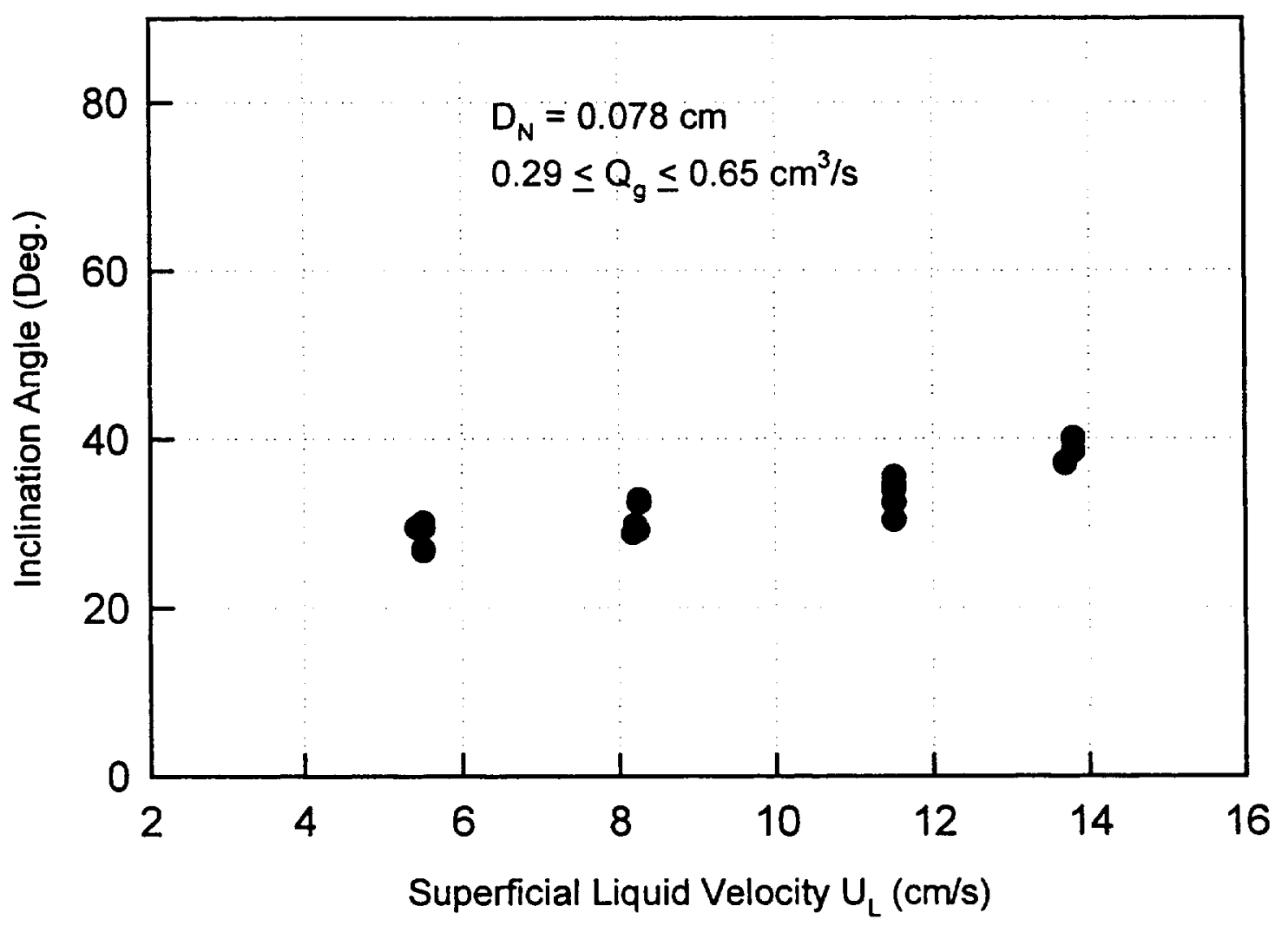

Fig. 14 . 


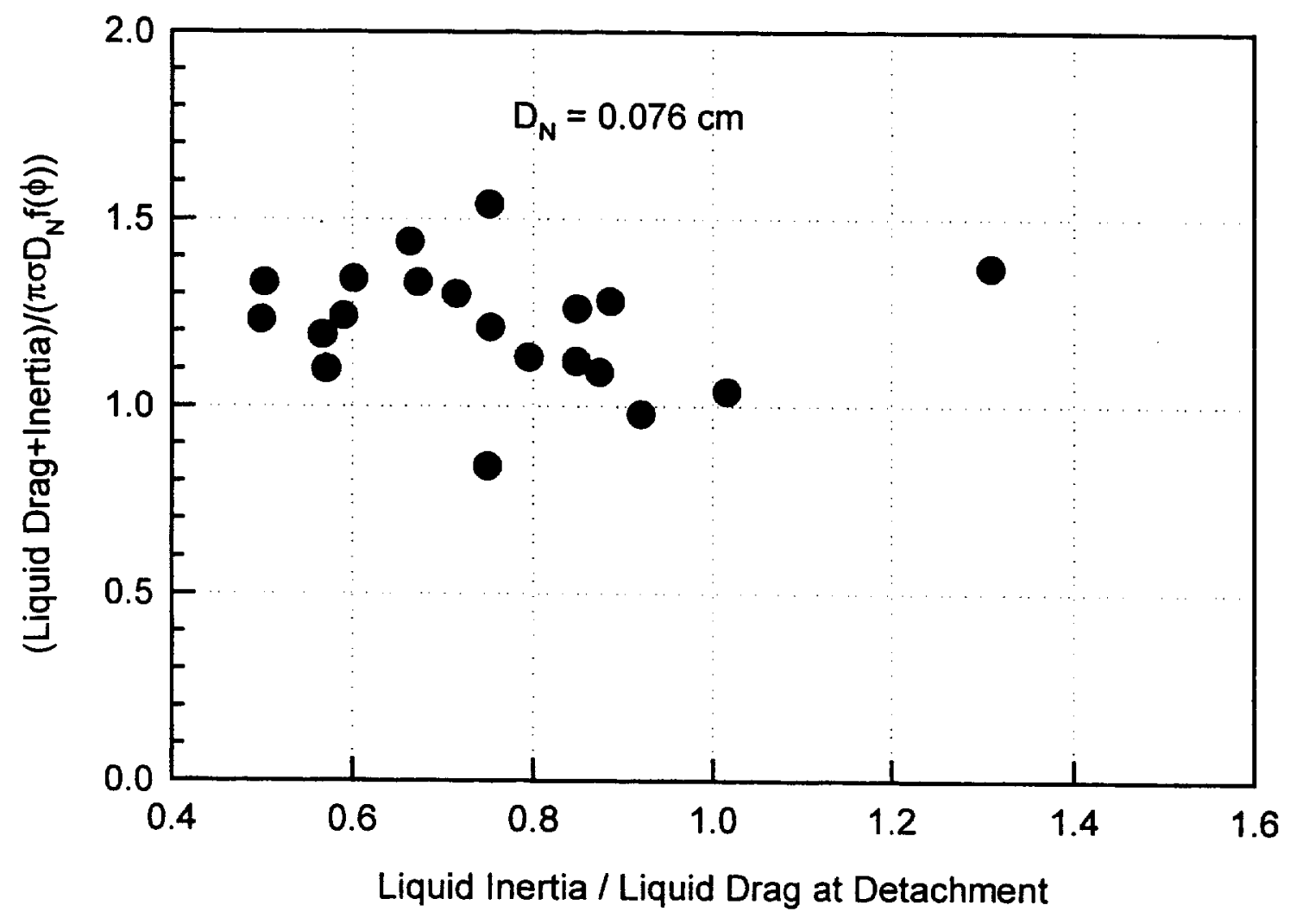

Fig. 15 . 